\title{
Nonlinear absorption spectra of ethidium and ethidium homodimer
}

Joanna Olesiak Banska ${ }^{1 *}$, Piotr Hanczyc ${ }^{1,2}$, Katarzyna Matczyszyn ${ }^{1}$, Bengt Norden ${ }^{2}$, Marek Samoc $^{1}$

${ }^{1}$ Institute of Physical and Theoretical Chemistry, Wroclaw University of Technology, Wybrzeze Wyspianskiego 27, 50-370 Wroclaw, Poland

${ }^{2}$ Department of Chemical and Biological Engineering, Chalmers University of Technology SE-41296, Gothenburg, Sweden

\section{Key words}

Z-scan, ethidium bromide, ethidium bromide homodimer, two-photon absorption, intercalation

* Corresponding author

joanna.olesiak@pwr.wroc.pl

\begin{abstract}
The Z-scan technique was used to determine the spectral dependence of the nonlinear absorption in well-known DNA intercalators: ethidium bromide and its homodimer. It is found that the compounds show essentially the same features of their nonlinear absorption spectra with the magnitudes of the relevant cross sections scaling with molecular weight of chromophore compound.
\end{abstract}

\section{Introduction}

In the late 1980-ties W. W. Webb and coworkers developed the technique of twophoton excited fluorescence microscopy (TPFM), which provided better fluorescence images [1] through the increase of contrast obtained by the removal of out-of-focus fluorescent light. Since then, TPFM is a technique of choice especially for imaging cells and other biological samples [2]. Spectral dependencies of two-photon absorption cross sections of several wellknown dyes, with possible use in this technique, were determined by $\mathrm{Xu}$ and Webb [3]. The 
widespread and increasing use of the technique has also led to intensive search for new materials with high two-photon absorption (2PA), and with efficient fluorescence and good photochemical stability. The focus was both on developing new dyes and investigating nonlinear optical properties of standard biological markers used in one-photon microscopies [3-5]. An important issue is to develop dyes that both exhibit high 2PA properties and have the ability of binding to specific biomolecules and/or emit bright fluorescence.

Among numerous nucleic acid stains, ethidium bromide (EB) (structure in inset to Fig.2). is one of the strongest DNA-binding dyes, with a dramatic increase of fluorescence, when intercalated to DNA [6]. The interaction between the dye molecule and DNA helix can be increased further by using dimeric molecules. The affinity of ethidium homodimer to nucleic acids is stronger than the affinity of EB (binding constants $K_{E B \text {-dimer }}=2 \times 10^{8} \mathrm{M}^{-1} ; K_{E B}$ $=1.5 \times 10^{5} \mathrm{M}^{-1}$ in $0.2 \mathrm{M} \mathrm{Na}^{+}$). However, the fluorescence quantum yield of the monomer, $\phi_{\text {monomer, }}$, is $\sim 1.8$ times higher than $\phi_{\text {dimer }}[7]$ due to a self-quenching effect.

EB and other dyes with phenanthridinium as chromophoric moiety, such as propidium iodide (PI), can be successfully applied in TPFM of biological samples. Recently, our group imaged self-assembled, condensed DNA structures using TPFM and DNA staining with PI and Hoechst 33342 [8-9]. Both dyes were found to exhibit high fluorescence and good stability under illumination with a focused femtosecond laser beam. Moreover, we were able to probe the local orientation of DNA strands in a sample volume thanks to a well-defined mode of binding of the dyes with DNA. Similar results can be expected for EB and EBhomodimer staining.

In order to efficiently excite the dyes, information about spectral dependence of 2PA cross-section, $\sigma_{2}(\omega)$, is required. $\sigma_{2}$ is a measure of probability of simultaneous absorption of two photons by a molecule, usually expressed in units of Goeppert Mayers, $1 \mathrm{GM}=10^{-50} \mathrm{~cm}^{4} \mathrm{~s}$ photon $^{-1}$ molecule $^{-1}$. In the case of fluorescent stains, $\sigma_{2}$ can be determined either from twophoton excited fluorescence [3] or directly by transmission measurements in the Z-scan technique [10]. The former approach was applied by Lakowicz and co-workers to evaluate $\sigma_{2}$ of EB intercalated to DNA. Assuming that the one-photon excited fluorescence quantum yield, $\phi$ is valid for the two-photon excitation case they obtained the $\sigma_{2}$ value equal to $6.7 \mathrm{GM}$ at $870 \mathrm{~nm}$ [11]. Similar experiments were performed for unbound PI [5]. The 2PA spectrum was presented, with 2PA maximum at $989 \mathrm{~nm}$, but without any quantitative description. The two-photon fluorescence technique requires a reference probe [12], careful determination of $\phi$ (with the assumption that one- and two-photon excited fluorescence occurs from the same 
level), and exclusion of one-photon excited fluorescence from the collected signal. On the other hand, the Z-scan technique can determine $\sigma_{2}$, also for non-fluorescent molecules and even in the range of wavelengths of excited state absorption [13].

In this report, we present the 2PA spectra of free EB and EB-dimer in solutions. The dispersion of $\sigma_{2}$ is determined with the fs Z-scan technique in a broad range of wavelengths, from 600 to $1550 \mathrm{~nm}$. The spectra are compared with one-photon absorption (1PA) spectra and the potential applications of both dyes in NLO microscopy are discussed.

\section{Materials and experimental methods}

\section{Chemicals and linear characterization}

EB and EB-dimer were purchased from Sigma Aldrich. $10 \mathrm{mg} / \mathrm{ml}$ solutions of EB and EB-dimer in water and DMSO, respectively, were prepared for Z-scan measurements. Linear absorption spectra were measured for appropriately lower concentration, with Thermospectronic Unicam UV 300 spectrophotometer.

\section{Z-scan measurements}

Two-photon absorption is a third-order nonlinear process, in terms of microscopic quantities it is related to the imaginary part of the cubic hyperpolarizability $\mathrm{g}$ of a molecule, but more usually it is expressed as the two-photon absorption cross section $\sigma_{2}$. The Z-scan technique[10] can be used to determine both the nonlinear refraction and nonlinear absorption of bulk samples, in the case of dyes the measurements are conveniently performed on solutions[14] placed in a cell that is made to travel along the focused Gaussian-shaped laser beam. Closed aperture (CA) and open aperture (OA) Z-scan traces can be recorded simultaneously in a single Z-scan run and used to determine the bulk nonlinear optical parameters of the solutions. In principle, recording just OA traces would be sufficient for the measurements of nonlinear absorption, however our experimental routine calls for recording both OA and CA traces. 130 fs pulses were delivered by a laser systems consisting of a Quantronix Integra-C regenerative amplifier operating as a $800 \mathrm{~nm}$ pump and a QuantronixPalitra-FS BIBO crystal-based optical parametric amplifier, with the repetition rate of $1 \mathrm{kHz}$. The investigated solutions were placed in $1 \mathrm{~mm}$ path length, stoppered glass cuvettes. The output from the Palitra was appropriately filtered using wavelength separators and color glass filters. It was then attenuated to microjoule per pulse energy range. The beam was focused to 
a focal spot of the beam waist $\mathrm{w}_{0}=25-60 \mu \mathrm{m}$ which resulted in peak intensities in the range from 60 to $150 \mathrm{GW} \mathrm{cm}^{-2}$. Three InGaAs photodiodes (Thorlabs) collected the reference signal, the OA signal, and the CA signal.

Each wavelength datapoint involved measurement on a $4.66 \mathrm{~mm}$ thick fused silica plate which provided the reference, a $1 \mathrm{~mm}$ glass cell filled with the solvent and identical cell with the solution. The data was analyzed as described in Ref.[15] to obtain microscopic nonlinear optical parameters of the dye molecules.

\section{Results and Discussion}

Figure 1 shows representative OA and CA Z-scan traces of EB-dimer at $775 \mathrm{~nm}$. CA traces show defocusing before and focusing after the focal plane of Z-scan setup, which is a standard pattern in case of positive nonlinear refractive index materials. The OA trace shows a decrease of transmittance at the focus, due to 2PA. The experimental points are well reproduced by the theoretical curves which justifies assumption that the absorption is due to a two-photon process.

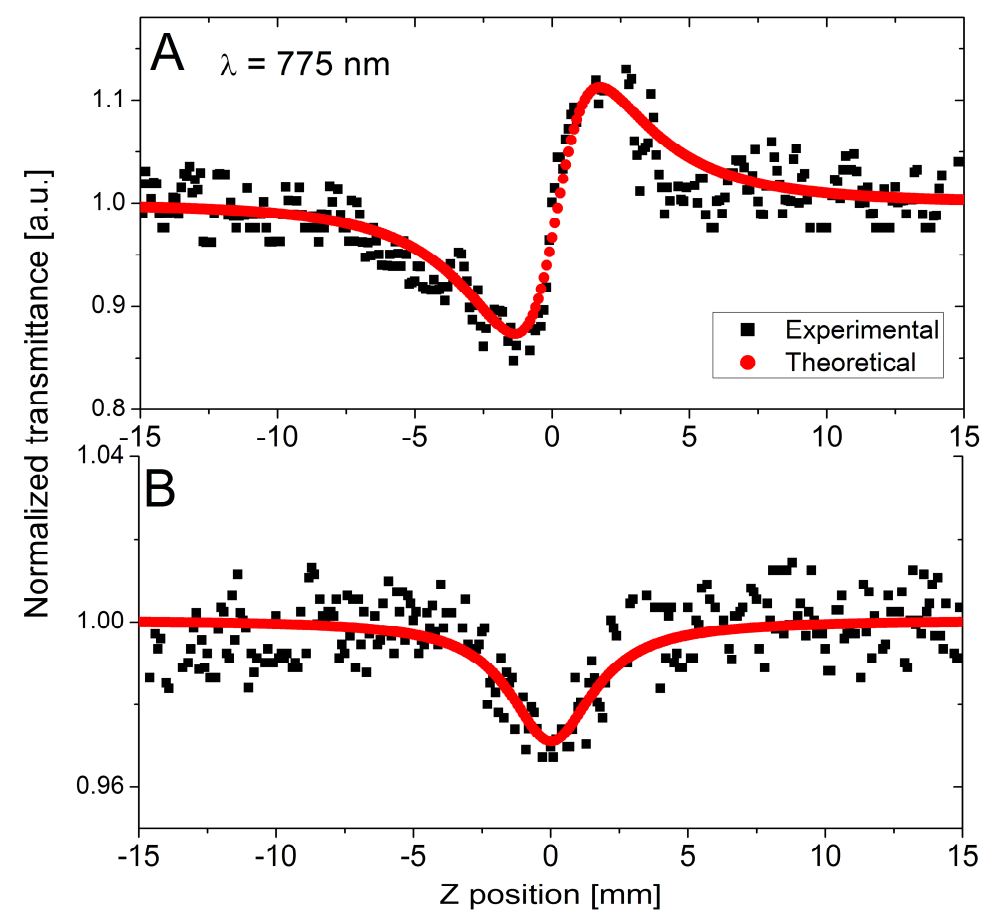

Fig.1. Representative CA (A) and OA (B) Z-scan traces and the theoretical fits for EB-dimer at $775 \mathrm{~nm}$ (1\% solution in DMSO). The CA trace was divided by the OA trace to obtain a purely refractive effect. 
For a few wavelength datapoints some contribution from three-photon absorption could not be excluded, however. For comparison the 2PA results are shown together with 1PA curves plotted at the doubled wavelength (see Fig.2. and Fig.3.). In both cases very strong $2 \mathrm{PA}$ is detected between $600 \mathrm{~nm}$ and $700 \mathrm{~nm}$. This range of wavelengths corresponds to two-photon excitation of the phenanthridinium part of the molecule (transition parallel to the long axis of EB molecule). The second absorption band is present at longer wavelengths, with a maximum at $950 \mathrm{~nm}$ and $1100 \mathrm{~nm}$ for EB and EB-dimer, respectively. In this region absorption involves the transition dipole moment directed along the phenyl-phenanthrene bond and as this part of the two molecules is different, the shift in the wavelengths is observed. 2PA cross-sections are equal to $8.2 \mathrm{GM}$ at $950 \mathrm{~nm}$ and $21.7 \mathrm{GM}$ at $1100 \mathrm{~nm}$ for EB and EB-dimer, respectively. The higher value of 2PA in case of the dimer is favorable from the applications point of view, e.g. in microscopy. The bisintercalation of two phenanthridinium moieties into DNA results in a stronger binding and more stable complexes with DNA under laser illumination. However, the straightforward comparison of $\sigma_{2}$ values may not give the clear answer which dye is more advantageous. Lately, different ways of scaling NLO merit were discussed [16-17]. Depending on the molecules different scaling factors were proposed: molecular weight, molecular volume, effective number of electrons [18] or even financial cost. In case of EB and EB-dimer, $\sigma_{2}$ normalized with molecular weight has similar values $\left(\sigma_{2} \mathrm{~EB} / \mathrm{M}=0.021, \sigma_{2}\right.$ EB dimer $\left./ \mathrm{M}=0.025\right)$. Phenanthridinium parts of both dyes occupy similar number of DNA binding sites, there is no possibility to attach more dimers to DNA, due to neighbour exclusion principle and steric hindrance. However, as was mentioned before, EB-dimer in complex with DNA is less fluorescent. Taking all the considerations into account EB can be suggested to be a better DNA marker in TPFM. When additional fluorescence polarization analysis is performed in order to determine the orientation of DNA [8-9], bisintercalated dimer moieties induce depolarization of fluorescence signal and make the analysis difficult. 

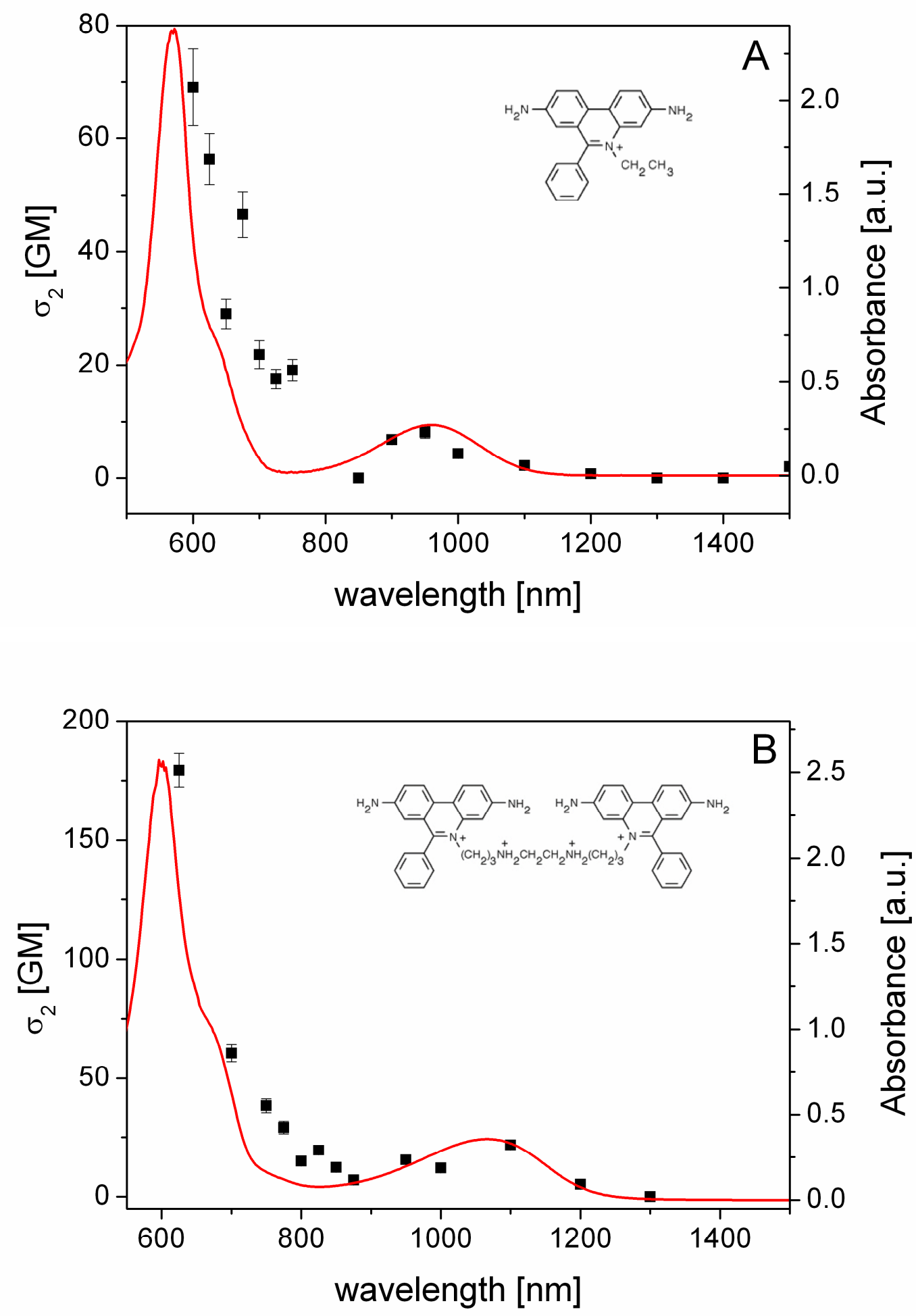

Fig.2. 1PA (red line) and 2PA (black squares) spectra of EB (A) and EB-dimer (B). One photon spectrum is plotted with the wavelength multiplied by a factor of two. Inset shows the EB and EB-dimer molecular structure. 


\section{Conclusion}

Two-photon absorption spectra of EB and EB-dimer were measured with the femtosecond Z-scan technique. Both dyes exhibit 2PA in the range of wavelengths $600 \mathrm{~nm}$ $800 \mathrm{~nm}$. The long wavelength $2 \mathrm{PA}$ maximum is located at $950 \mathrm{~nm}$ and $1100 \mathrm{~nm}$, with $\sigma_{2 \mathrm{~EB}}=$ $8.1 \pm 1 \mathrm{GM}$ and $\sigma_{2 \mathrm{~EB} \text { dimer }}=21.7 \pm 1.5 \mathrm{GM}$ for EB and EB-dimer, respectively. $\sigma_{2}$ normalized with molecular weight has similar values for both dyes. This is the first time when 2PA spectrum of EB-dimer in a broad wavelength range is presented and compared with 2PA of unbound EB. Further research is ongoing on NLO properties of other dimeric dyes in polymer self-standing films with DNA [19], as a new material with potential application in biology.

\section{Acknowledgements}

We gratefully acknowledge funding for this research from the Foundation for Polish Science, Programme "Welcome". PH and BN acknowledge financial support from European Research Council (ERC) - Senior Advanced Grant.

\section{References}

[1] W. Denk, J.H. Strickler, W.W. Webb, Science, 248 (1990) 73-76.

[2] W.R. Zipfel, R.M. Williams, W.W. Webb, Nat Biotechnol, 21 (2003) 1368-1376.

[3] C. Xu, W.W. Webb, J Opt Soc Am B, 13 (1996) 481-491.

[4] M. Pawlicki, H.A. Collins, R.G. Denning, H.L. Anderson, Angew Chem Int Edit, 48 (2009) 3244-3266.

[5] F. Bestvater, E. Spiess, G. Stobrawa, M. Hacker, T. Feurer, T. Porwol, U. BerchnerPfannschmidt, C. Wotzlaw, H. Acker, J Microsc-Oxford, 208 (2002) 108-115.

[6] J. Olmsted, D.R. Kearns, Biochemistry-Us, 16 (1977) 3647-3654.

[7] B. Gaugain, J. Barbet, N. Capelle, B.P. Roques, J.B. Lepecq, M. Lebret, Biochemistry-Us, 17 (1978) 5078-5088.

[8] J. Olesiak-Banska, H. Mojzisova, D. Chauvat, M. Zielinski, K. Matczyszyn, P. Tauc, J. Zyss, Biopolymers, 95 (2011) 365-375.

[9] H. Mojzisova, J. Olesiak, M. Zielinski, K. Matczyszyn, D. Chauvat, J. Zyss, Biophys J, 97 (2009) 2348-2357.

[10] M. Sheik-Bahae, A.A. Said, T.H. Wei, D.J. Hagan, E.W. Van Stryland, IEEE Journal of Quantum Electronics, 26 (1990) 760. 
[11] H. Malak, F.N. Castellano, I. Gryczynski, J.R. Lakowicz, Biophys Chem, 67 (1997) 3541.

[12] N.S. Makarov, M. Drobizhev, A. Rebane, Opt Express, 16 (2008) 4029-4047.

[13] L.A. Padilha, S. Webster, H.H. Hu, O.V. Przhonska, D.J. Hagan, E.W. Van Stryland, M.V. Bondar, I.G. Davydenko, Y.L. Slominsky, A.D. Kachkovski, Chem Phys, 352 (2008) 97-105.

[14] M. Samoc, A. Samoc, B. Luther-Davies, M.G. Humphrey, M.S. Wong, Opt Mater, 21 (2003) 485-488.

[15] M. Samoc, A. Samoc, G.T. Dalton, M.P. Cifuentes, M.G. Humphrey, P.A. Fleitz, Twophoton absorption spectra and dispersion of the complex cubic hyperpolarizability $\gamma$ in organic and organometallic chromophores, in: I. Rau, F. Kajzar (Eds.) Multiphoton Processes in Organics and Their Application, Old City Publishing, Philadelphia, 2011, pp. 341-355.

[16] R.L. Roberts, T. Schwich, T.C. Corkery, M.P. Cifuentes, K.A. Green, J.D. Farmer, P.J. Low, T.B. Marder, M. Samoc, M.G. Humphrey, Adv Mater, 21 (2009) 2318-+.

[17] T. Schwich, M.P. Cifuentes, P.A. Gugger, M. Samoc, M.G. Humphrey, Adv Mater, 23 (2011) 1433-1435.

[18] M.G. Kuzyk, J Chem Phys, 119 (2003) 8327-8334.

[19] P. Hanczyc, B. Norden, B. Akerman, J Phys Chem B, 115 (2011) 12192-12201. 